\title{
Distributed power control and beamforming for cognitive two-way relay networks using a game-theoretic approach
}

\begin{abstract}
This paper studies a cognitive two-way relay network in which multiple pairs of secondary users (SUs) exchange information with the help of multiple relays. We propose a distributed power control and beamforming algorithm that enables the users operating in the underlay mode to strategically adapt their power levels, and maximize their own utilities subject to the primary user (PU) interference constraint, as well as its own resource and target signal-tointerference-and-noise-ratio (SINR) constraints. The strategic competition among multiple decision makers is modeled as a non-cooperative game where each secondary user (SU) acts selfishly in the sense of maximizing its own utility. An adaptive method is proposed to determine appropriate pricing function. The problem of beamforming optimization under amplify-and-forward (AF) protocol is addressed as a generalized eigen value problem with respect to the utility function of SUs. The existence of a unique Nash equilibrium (NE) is proved and several numerical simulations are conducted to quantify the effect of various system parameters on the performance of the proposed method.
\end{abstract}

Keyword: Two-way relay networks; Distributed power control; Beamforming; Game theory 